\title{
REITER'S SYNDROME IN CHILDHOOD
}

\author{
BY \\ BERYL D. CORNER \\ From the Department of Child Health, Bristol University, and Bristol Royal Hospital for Sick \\ Children
}

(Received for Pubuication february 6, 1950)

Since Reiter (1916) gave his name to the syndrome characterized by arthritis, conjunctivitis, and urethritis, extensive reports of this interesting condition have appeared in the world literature.

A study of the reported series of cases reveals certain outstanding features. Until the last few years these cases all consisted of young adult males, although before Reiter's paper several authors noted joint symptoms in women, with conjunctivitis or vaginitis (Huette, 1869; Gauster, 1869; Krāuter, 1871). Vallée (1946), in reporting a series of 151 male cases, quoted the youngest recorded case of a boy aged 16 years (Junghanns, 1918) and an arthritic case in a woman (Lever and Crawford, 1944). In the last three years, three cases have been reported in children under 12 years of age, and this diagnosis has been suggested in two younger brothers of a reported case in a boy of 15 years. Paronen (1948), in a report of 344 cases in Finland, gives the most comprehensive survey yet made and states that $10 \%$ of his patients were women. $\mathrm{He}$ describes a case in a child aged 2 years and mentions that three other patients were aged 12 to 15 years.

The very close association of the condition with Flexner dysentery has been stressed by most authors and, in fact, post-dysenteric arthritis was well known before Reiter's description of the syndrome. Paronen's series of cases occurred during the autumn of 1944, following a severe epidemic of Flexner dysentery in the Karelian Isthmus, and in $96.4 \%$ of his cases was there a previous Flexner infection, two-thirds of the cases occurring from 11 to 30 days after the onset of the dysentery.

All three cardinal symptoms occur in most of the recorded cases, but arthritis seems to predominate and is frequently the first sign. Involvement of the large weight-bearing joints of the lower extremity is usual, the knee joint being most commonly affected and then the ankle, but in nearly all cases it is a polyarticular arthritis. In Paronen's series $97 \cdot 3 \%$ of cases showed articular manifestations and in $42 \%$ of these the knee joints were affected. Vallée also stresses this articular incidence. In some cases the lesions are so slight as to consist of migratory pains in the joints only, but in a large number of cases considerable effusions developed; bursae and tendon sheaths may also be closely related to the joint lesions. Bone destruction is usually very slight and occurs late in the disease (Twiss and Douglas, 1946). The occasional sequel of ankylosis has been noted by a number of writers, Vallée (1946), and Hollander, Fogarty, Abrams, and Kydd (1945).

The most common ocular manifestation of the syndrome is conjunctivitis, either uni- or bilateral, but involvement of the iris, uveal tract, and cornea has been reported by Vallée, Zewi (1947), and others. The degree of conjunctivitis varies from mild cranberry colour redness and velvety appearance to severe purulent conjunctivitis. The eye manifestations frequently occur early in the disease and often coincide with the onset of arthritis. The incidence of conjunctivitis varies considerably in different series of cases: Dorendorf (1917), 25.5\%; Walther (1941), 4.5\%; and Paronen (1948), $8 \%$.

Involvement of the urinary tract is considered to be the third common manifestation. Urethritis is the usual lesion, but cystitis, prostatitis, and nephritis have all been noted by many writers (Vallée, Hollander, and others). The urethritis is frequently extremely mild, being little more than the complaint of a burning sensation on micturition in many cases. Where actual discharge occurs it is often seropurulent and much less profuse than in gonorrhoea. Paronen noted involvement of the urogenital organs in $79.3 \%$ of his cases, with urethritis in $90 \%$ of these. Renal lesions were found in nine of his patients. Urethritis may occasionally initiate the syndrome but it usually accompanies one of the other essential symptoms.

Generalized lymphadenitis has been noticed in many cases, with enlargement of the spleen occasionally. Skin lesions on the genitalia and over the affected joints have also been recognized; pleurisy and cardiac lesions have rarely been noted.

The course of this disease varies from two to five months and recovery is the rule, although 
recurrences have been reported; in Kardung's (1943) case these lasted over a period of 15 years.

Many types of treatment have been attempted, including salicylates, arsenicals, pyrotherapy, sulphonamides and antibiotics, but without noticeable effect in a disease which is, in any case, self-limited, although recently Warthin (1948) gives an encouraging report of the use of streptomycin.

The aetiology of the condition has provoked much interest. The outstanding feature that all pus and synovial fluids are sterile has suggested the possibilities of virus infections and allergy.

Dienes and Smith (1942) have, however, isolated the pleuropneumonia bacillus from urethral discharge and synovial fluid of a small number of patients. These organisms have also been isolated from vaginal discharge in women and in cases of non-specific urethritis in men (Beveridge, 1943).

The relationship with bacillary dysentery, however, appears to be absolutely proved by a large number of observers, and the occurrence of diarrhoea or proved dysentery from 10 to 30 days preceding Reiter's syndrome led Walther (1941) to consider that it was an allergic manifestation and not merely a complication of dysentery. Paronen goes so far as to state that on the basis of his investigations Reiter's disease seems to occur only after dysenteric infection, proof of the infection being obtained if necessary by serological means only, where no dysentery infection can be proved directly.

In a discussion of Reiter's syndrome, mention must be made of keratosis blenorrhagica, the clinical entity characterized by urethritis, polyarthritis, and skin lesions. The latter present a typical picture of vesicles leading to crust formation and the development of horny masses, which occur particularly on the trunk, extremities, including the palms and soles, and the buccal mucous membranes. The earlier papers dealing with this syndrome considered that it was essentially associated with gonorrhoea; Lees and Percival (1931) give a full report of the condition and consider the possibility of hypersensitivity to gonococcal toxin. Recently the similarity to Reiter's syndrome has been pointed out by Epstein (1947), and the absence of proof of gonococcal infection in many cases suggested the same aetiology for the two syndromes. Peters (1949) suggests that keratosis blenorrhagica is essentially the same condition as Reiter's syndrome, although the rarity of cases with skin lesions in epidemics of Reiter's syndrome is notable.

There are two recorded cases of keratosis blenorrhagica in childhood. The first is described by Robert (1897) in a child aged 4 years, and the second by Gault and Gault (1948) in an Indian girl of 7 years. In the latter case the skin lesions were typical but there was no definite evidence of urethritis or arthritis.

The first recorded case of classical Reiter's syndrome in a child appears to be in a series of 10 cases reported by Zewi (1947). A boy aged 4 years had an attack of diarrhoea, followed after one day by conjunctivitis which became bilateral. He was admitted to the Ophthalmic Clinic of the University of Turku. A week later there was dysuria with albumin and leucocytes in the urine. On the seventeenth day after the onset of diarrhoea swelling of a metacarpo-phalangeal joint was noted, followed by arthritis in one elbow and the knee joints. Bilateral keratitis occurred. The patient was free from symptoms in just over two months. No organisms were cultured from urine or conjunctival swabs. There is no note of faecal cultures or blood agglutination tests for dysentery.

This author also reports a typical case in a boy aged 14 years and in two girls aged 15 and 16 years.

In 1946, however, Koster and Jansen record a Dutch family in which the eldest child, aged 15 years, had diarrhoea, followed after a week by purulent bilateral conjunctivitis, dysuria, and slight polyuria on the following day. Shortly after these symptoms there followed a painful swelling in his left ankle joint and big toe. The urinary symptoms only lasted for one week, but swelling of the tarsus and first metatarso-phalangeal joints lasted for some weeks. No pathogenic organisms were isolated from conjunctival swabs. A brother aged 3 years, who also had diarrhoea at the same time, developed conjunctivitis and urethritis eight days later, but no articular manifestations; and a third boy, aged 11 years, also had symptoms of urethritis, but these two younger children had recovered before medical attention was sought for the eldest boy. In all the boys the blood showed a positive agglutination to Sh. flexneri but the type and height of titre is not recorded. They would, however, all appear to be cases of Reiter's syndrome in varying degrees of severity. All cleared up without specific treatment and with no sequelae.

Florman and Goldstein (1948) record a white male child admitted to Mount Sinai Hospital, New York, in August, 1947. He had watery diarrhoea, followed five days later by urgency, dysuria, and frequency of micturition. Two days later there was pyrexia, swelling of the right knee, and bilateral purulent conjunctivitis. This child also presented generalized lymphadenopathy and a cardiac systolic murmur at the apex and left sternal border, with no cardiac enlargement. Greenish-yellow pus was aspirated from the joint. His urinary symptoms 
subsided in a week, but he had an exacerbation of conjunctivitis with keratitis in the right eye occurring on the fifteenth day of illness. Two months later the knee joint was completely normal and the only residual abnormality was a small corneal opacity. Penicillin therapy was given but the keratitis actually developed during the course of this. Many investigations were done on the patient. The only organisms recovered were a staphylococcus albus from the conjunctivae and a chromogenic bacillus from the knee fluid. No evidence of virus disease was found after inoculation of rabbit cornea with knee fluid and urethral discharge; also mice were inoculated with fluid from the knee joint intracerebrally and intraperitoneally. The only serological tests giving a positive result were those for Sh. flexneri vii and viii. The titre for Sh. flexneri vii fell from 1:1,280 in the second week of the disease to $1: 40$.

Paronen gives details of a case occurring in Finland in a boy aged 2 years 8 months, who developed the syndrome in August, 1946. In common with other members of his family, this child developed fever and diarrhoea, with blood in the stools, lasting for two days. After nearly a fortnight he developed bilateral conjunctivitis, followed three days later by purulent urethritis, and tenderness and swelling of both knees and ankles and the left elbow. Three weeks later his blood agglutination showed Flexner $A+D-W x$ positive at 1:80. Cultures from urine and stools showed no pathogens and his symptoms cleared completely within four months. Paronen also mentions that three of his other cases were boys aged 12 to 15 years, but no clinical details of these cases are given, although it may be inferred from his paper that all had been preceded by dysentery.

Thus, all previously recorded cases in children appear to be typical in that diarrhoea preceded the onset of symptoms by 5 to 14 days, and the cardinal triad of symptoms was present in two of the cases under 12 years. The association with Flexner dysentery infection also seems present in all these children. Although the initial symptoms were relatively severe, the clinical course has been typical, with fairly rapid recovery compared with cases reported in adults. It may also be noted that all cases in childhood have been recorded in males.

The following account of this syndrome occurring in a boy aged 9 years is believed to be the first reported in Britain in a child, and has an additional interest in the coincident finding of a congenital abnormality of the urinary tract which does not appear to have been previously noted in recorded series of cases.

\section{Case Report}

Piers M., age 9 years, was admitted to Bristol Children's Hospital on November 19, 1948, complaining of pain in the right hip.

PAST History. A premature baby (birth weight $4 \frac{1}{2} \mathrm{lb}$.) he was breast-fed and made average progress. His previous illnesses were measles, pertussis, varicella, and tonsillectomy at 5 years. No history of gastro-intestinal disorder could be obtained.

Family and Social History. The patient is the first child in the family. His parents separated shortly after he was born and he remained with his mother, who subsequently remarried and had two more children who are healthy. His mother is a severe asthmatic, frequently completely incapacitated; her three siblings and mother also had asthma or hay fever. The father was a healthy man. The family lived in a condemned basement flat in Clevedon, Somerset. The stepfather, a commercial traveller, was away from home a great deal, so that owing to the mother's frequent incapacity a considerable amount of domestic work and responsibility was left to the child, who received minimal care himself. No history of dysentery or any recent alimentary tract disorder in the relatives could be obtained.

History of Present Illness. Three weeks before admission the child experienced soreness and difficulty in micturition, accompanied by marked frequency, particularly at night. There was slight pyrexia up to $100^{\prime}$ F. A course of sulphamezathine, g. $0 \cdot 5$ four-hourly for five days, was given by the general practitioner and the symptoms improved considerably, dysuria ceasing after a few days, but frequency persisting. Two weeks later bilateral mild conjunctivitis appeared, with watery discharge. This was considered to be similar to that occurring in epidemic form at the time in the neighbourhood. He also had mild pain in the right buttock but was able to walk about as usual. Two days before admission he had severe pain in the right hip region which woke him frequently at night, caused him to limp, and eventually forced him to remain in bed.

EXAMINATION (November 19, 1948.) The patient appeared an intelligent, cheerful child, of average height for his age, weighing $57 \mathrm{lb}$., thin, with moderate pallor of mucous membranes. The temperature was $99^{\circ}$, pulse 100 , respirations 20 . There was acute inflammation of both conjunctivae, with some oedema of the palpebral conjunctiva and increased lachrymation; no pus was seen. There was no obvious infection in the ears, nose or throat and the cervical glands were just palpable in the anterior and posterior triangles. The right lower limb was held in adduction, with slight flexion of the hip joint. There was tenderness on pressure over this joint and some limitation of all movements. There was no abnormality of heart, lungs or abdomen, and no obvious urethral discharge. Blood pressure: 10060 . Urine: specific gravity 1012, alkaline; albumen, heavy cloud; large quantities of pus, no organisms.

Course. During the first week in hospital, with bed rest only, the physical signs in the hip joint cleared up and full range of movement was obtained on the fifth day after admission. The temperature settled to 
normal. On November 24 an effusion developed in the right knee joint causing considerable heat, pain, and swelling: this was followed 48 hours later by similar signs in the left knee. There was a slight rise of temperature, the range varying between $98^{\circ}-100^{\circ}$. Sodium salicylate, gr. 60 daily, was then given for 10 days with no apparent effect on the joint lesions or pyrexia. Following this the child looked more ill, became less cheerful, and his appetite was bad.

On December 6 the right ankle became swollen and tender and pyrexia increased up to $101 \cdot 8^{\circ}$ on December 9. Intermittent pyrexia continued for the following week and the child's condition appeared to be deteriorating. He looked pale, toxic, and lost $4 \frac{1}{2} \mathrm{lb}$. in weight. Throughout the month of December there was little change in the knee joints or general condition. From December 28 to January 8, 1949, he had another bout of pyrexia with maximum temperature $102 \cdot 4^{\circ}$. He appeared to have a slight coryza during this time but no pathogenic organisms could be cultured from nose or throat swabs.

On January 6 his weight had dropped to $48 \mathrm{lb}$. There was still swelling (Fig. 1) of both knees and the right ankle joints, but he was now afebrile and remained so throughout the rest of $h$ is illness. Marked wasting had developed in the thigh muscles and lymphatic glands were now enlarged in the left supraclavicula $r$ region, both axillae and groins. The spleen was never palpable. He was then given a ten-day course of penicillin, with total dosage of $2,400,000$ units in four-hourly intramuscular injections, since on this occasion only, Staphylococcus aureus was cultured from the urine. Throughout this time he continued to have very marked frequency of micturition with occasional pain and persisting albumen and pus in the urine. His conjunctivitis had cleared up completely. Throughout January he appeared to improve slightly and began to gain weight.

During February his frequency ceased, the quantity of albumen and pus in the urine suddenly diminished, and the joint swellings became much less. On February 23 he complained of pain in the left ankle for the first time and this was noted to be swollen.

During March the condition of all affected joints appeared to improve very much and full range of movement returned. He gained $5 \mathrm{lb}$. in weight. Early in April he started to get up and was soon walking well. The urine was now quite normal. At the end of April there was slight recurrence of swelling in the right knee joint but this settled again after a week's rest in bed.
He was discharged to a convalescent home for three months at the end of June, 1949, and was last seen in December, 1949, when he was free from symptoms, appeared well, and the only abnormality to be detected was slight residual thickening of the knee joints.

Investigations. From November 19 to December 31, 1948, the following investigations were carried out:

URINE. A larger number of specimens of urine were examined. All contained a heavy cloud of albumin, large numbers of pus cells, no blood or casts and were sterile on culture. Several twenty-four-hourly specimens were examined and cultured for tubercle bacilli, with negative results.

Blood Sedmentation Rate (Westergren Method). $80 \mathrm{~mm}$. in first hour on November 22, 1948. This rose to $110 \mathrm{~mm}$. in the first hour by December 30,1948 .

BloOD Counts. On November 23, 1948, haemoglobin $88 \%$ (Haldane), red cells $4,410,000$ c.mm., leucocytes $13,500 \mathrm{c}$. mm. (neutrophils $66 \%$, lymphocytes $18 \cdot 5 \%$, eosinophils $11 \%$, monocytes $3 \cdot 5 \%$, basophils $1 \% ; 20 \%$ of the neutrophils were 'juvenile forms with a general marked shift to the lef $t)$. Platelets normal.

On December 14, 1948, haemoglobin $75 \%$, red cells $3,530,000$ c. mm., leucocytes $12,600 \mathrm{c}$. $\mathrm{mm}$. (neutrophils $58^{\circ}$ o, lymphocytes $30^{\circ}$, eosinophils $3^{\circ}$, monocytes $9^{\circ}{ }_{0}$ ).

On December 30 , 1948, haemoglobin $66^{\circ}$ o, red cells $3,810,000$ c.mm., leucocytes 13,500 c.mm. BLOOD. Wassermann and Kahn tests negative. Gonococcal complement fixation test negative on December 22, 1948.

Conunctival Swabs. Several swabs were examined and all were sterile. Scanty pus cells were seen in one smear.

Nose and Throat Swabs. These were examined on November 29, 1948, and December 9, 1948. No pathogenic organisms were cultured.

EXAMINATION OF FaECES. All stools appeared normal. There was no microscopic blood or pus and three cultures showed no pathogenic organisms.

URETHRAL SMEAR after prostatic massage (December 16, 1948) showed many pus cells but culture was sterile.

On December 14, 1948, the right knee joint was aspirated. Blood-stained fluid was withdrawn, and on standing a large fibrin clot formed. Films showed red cells in large numbers and scanty leucocytes, $50 \%$ lymphocytes, and $50 \%$ neutrophils. No organisms were seen and culture was sterile.

Mantoux Test. $1 / 1,000$ and $1 / 100$ dilutions negative. 
During January, 1949, the following further investigations were made:

URINE. This was repeatedly examined and continued to contain large numbers of pus cells. On this occasion (January 3) only there was a moderate growth of coagulase negative, penicillin-sensitive Staphylococcus aureus, hence a course of penicillin intramuscularly was given. Subsequent cultures were all sterile. Urine, urethral, and conjunctival swabs were cultured for pleuropneumonia-like organisms with negative results.

Blood COUNT (January 24). Haemoglobin $73 \%$, red cells $3,660,000$ c.mm., leucocytes 7,800 -normal distribution.

Blood Sedimentation Rate. Remained at $100 \mathrm{~mm}$. in the first hour throughout the month.

BloOd UREA (January 24). $30 \mathrm{mg}$. ${ }^{\circ}$.

Blood Agglutination Reactions. These were performed on January 24 with the following results:

Agglutinations for suspensions of S. typhi (H.O.), S. paratyphi A(H.O.), S. paratyphi B(H.O.), Br. abortus, Br. melitensis, Sh. sonnei were negative.

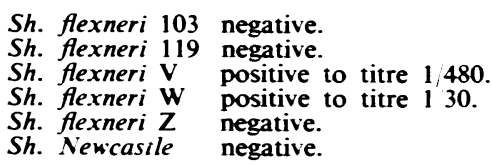

The agglutination test for Sh. flexneri $\mathrm{V}$ and $\mathrm{W}$ was repeated on the same batch of suspensions and with a fresh batch with the same results.

In view of the continuing pyuria, an intravenous pyelogram was performed. This (Fig. 2) showed a normal right renal pelvis and ureter, but on the left there were two pelves and a double ureter which appeared to fuse opposite the level of the fifth lumbar vertebra. Slight dilatation of the upper renal pelvis was suggested. As a result of these findings, cystoscopy and retrograde pyelography was performed by Mr. Wilfrid Adams on January 4. The bladder showed no ulceration but only

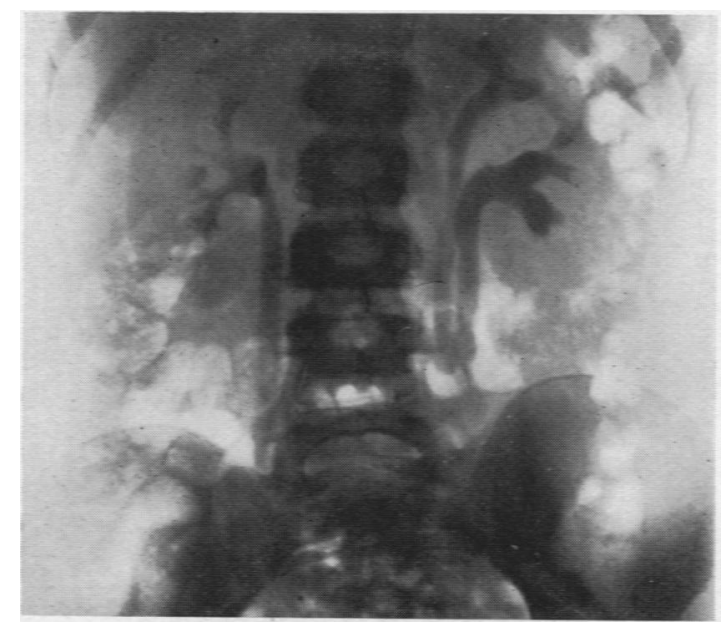

Fig. 2.-Intravenous pyelogram showing double left renal pelvis and ureter. two ureteric orifices were seen. These were catheterized and retrograde pyelography performed. The left ureteric catheter was passed easily into the upper renal pelvis which was clearly defined by pyelography; the lower left pelvis and second ureter did not fill with the opaque medium.

From the beginning of February the pathological tests all coincided with the rapid clinical improvement in the patient. On February 4 the urine contained no albumen, and only scanty pus cells (2-3 per high power field). This improvement has been maintained without any relapses since that date.

Blood sedimentation rate (February 14) had fallen to $32 \mathrm{~mm}$. in the first hour, 87 in the second hour. This continued to fall steadily, and on April 2 was $11 \mathrm{~mm}$. in the first hour and 35 in the second hour: by the end of April it was completely within normal limits.

A radiograph of the knee and ankle joints taken on February 15 showed osteoporosis of the bones adjacent to the joints, but no increase in joint space.

Blood counts showed a steady improvement of the anaemia: on March 17 haemoglobin $90^{\circ}$, red cells $4,550,000$ c.mm., leucocytes 10,650 c.mm. with normal distribution.

Blood serum on April 11 gave no agglutination reaction to Sh. flexneri $\mathrm{V}, \mathrm{W}$, and $\mathrm{Z}$.

Enquiry from the Medical Officer of Health for Clevedon rural district revealed that there had been no notified cases of Flexner dysentery in the district in 1948, and local general practitioners confirmed that there had been no epidemic gastro-enteritis.

\section{Discussion}

Reiter's syndrome in childhood appears to be a rarely noted condition, although it seems possible that where the incidence is high in the adult population of a community, cases of a mild nature may occur simultaneously among the children. These cases may present only one or two of the classical symptoms and the joint lesions may be extremely transitory. The close relationship of the syndrome to Flexner dysentery suggests that where an epidemic of dysentery has been known to occur in a district, during the following month careful observation of the child population may reveal a number of these milder cases.

All previously reported cases in childhood are quite typical, although of a relatively mild variety. The present case shows the classical picture of the syndrome and is of a rather more severe type and longer duration than these other cases. Urethritis was the first symptom in this patient, followed by conjunctivitis, and fairly severe arthritis affecting the lower extremities only. The duration of the disease was five months, during the whole of which time the erythrocyte sedimentation rate was raised and joint lesions were present. The urinary infection lasted for three months and was extremely severe. As in all previously recorded cases, the outstanding 
feature was the very large quantity of pus in the urine which was repeatedly sterile on culture. The dramatic clearance of the heavy pyuria and marked frequency during the course of only one week in the thirteenth week of the disease was striking. The finding of a congenital abnormality of the urinary tract is of some interest, but stasis of urine could not definitely be confirmed and there is no evidence that this abnormality influenced the course of the disease. The conjunctivitis in this case was extremely mild, although some excess redness of the conjunctivae was noted for about three months.

The development of a normocytic anaemia, with haemoglobin reduced to $66 \%$, during the first two months of the disease is in agreement with the findings of Paronen for $29.7 \%$ of his patients. He also noted a leucocytosis of 10,000 to 18,000 per c.mm. in $15.8 \%$ of his patients, with eosinophilia in $33 \%$. In this case there was a definite slight neutrophil leucocytosis and an eosinophilia (11\%) in the first blood count. It is to be noted that, coinciding with the rapid clinical improvement at the end of the third month, the blood count returned to normal limits without any special treatment.

This case throws no particular new light on the aetiology of the syndrome. It is typical in that no organisms could be cultured from discharges, joint fluid or excreta, and a search for pleuropneumonialike organisms gave negative results. The point of special interest is the significantly high agglutination titres for Sh. flexneri $\mathrm{V}$ at the height of the disease; these disappeared as the condition cleared up. It, therefore, seems likely that this child had a sub-clinical infection with Sh. flexneri $\mathrm{V}$ despite the absence of any history of alimentary tract disorder, and that this case is, therefore, exactly comparable to previously recorded cases in children in its close association with Flexner infections. The patient came from a highly allergic family, and the increase in eosinophils in the first blood count is compatible with an allergic reaction.

The suggestion that this condition may be due to a virus infection would seem unlikely in view of the large amount of pus produced in the urinary tract, since, as a general rule, virus infections are not associated with much pus production. In one of the recorded cases, that of Florman and Goldstein (1948), the fluid aspirated from a joint was definitely purulent. Therefore, while it seems unlikely that a virus is the primary agent in these cases, consideration might be given to the suggestion that some such infection, in association with Flexner dysentery, produces this very characteristic clinical syndrome.

Before diagnosis, one course of sulphamezathine and a course of salicylates were given, but as soon as diagnosis seemed certain the only other treatment was one course of penicillin in view of the numerous reports in the literature of the failure of all forms of treatment. This case is, therefore, typical in that the child recovered spontaneously and one year after the onset of the disease he is well and has had no relapses.

\section{Summary}

A review of the literature of the many series of cases of Reiter's syndrome shows that the condition has been rarely reported in childhood. An account of the only three other cases reported in detail in children under the age of 12 years has been given, and the relationship to dysentery indicated.

A typical, fairly severe case in an English boy of 9 years is presented, in whom, despite the absence of diarrhoea, a significantly raised serum agglutination titre for Sh. flexneri $\mathrm{V}$ was found, which fell to normal as the clinical condition recovered. In addition, the patient had a congenital abnormality of the urinary tract.

A suggestion is made that, following epidemics of dysentery, this condition may be found less rarely in childhood if a search is made for minimal clinical manifestations.

\section{REFERENCES}

Beveridge, W. I. B. (1943). Med. J. Aust., 2, 479.

Dienes, L., and Smith, W. E. (1942). Proc. Soc. exp. Biol., N.Y., 50, 99.

Dorendorf (1917). Med. Klinik., 13, 519.

Epstein, E. (1947). Arch. Derm. Syph., Chicago, 56, 191.

Florman, A. L., and Goldstein, H. M. (1948). J. Pediat., 33, 172.

Gault, E. W., and Gault, E. I. (1948). Indian med. Gaz., 83, 123.

Gauster, M. (1869). Abstract in Jber. Leist. ges., Med., 2, 147

Hollander, J. L., Fogarty, C. W., Abrams, N. R., and Kydd, D. M. (1945). J. Amer. med. Ass., 129, 593.

Huette (1869). Arch. gén. Méd., sér., 6, 14, 129.

Junghanns, O. (1918). Disch. med. Wschr., 44, 1304.

Kardung. Quoted by Stoia, I. (1943). Z. Rheumaforsch., 6, 168.

Koster, M. S., and Jansen, M. (1946). Ned. Tijdschr. Geneesk., 90, 483.

Kräuter, J. (1871). Abstract in Jber. Leist. ges. Med., 2. 158 .

Lees, D., and Percival, G. H. (1931). Lancet, 2, 1116.

Lever, W. F., and Crawford, G. M. (1944). Arch. Derm., Syph., Chicago, 49, 389.

Paronen, I. (1948). Acta med. scand., 131, Suppl. 212. Peters, J. H. (1949). Arch. Derm. Syph., Chicago, 59, 217.

Reiter, H. (1916). Dtsch. med. Wschr., 42, 1535.

Robert (1897). Ann. Derm. Syph., Paris, 16, 745.

Twiss, J. R., and Douglas, A. H. R. (1946). Ann. intern. Med., 24, 1043.

Vallé, B. L. (1946). Arch. intern. Med., 77, 295.

Walther, G. (1941). Münch. med. Wschr., p. 381.

Warthin, T. A. (1948). Amer. J. Med., 4, 827.

Zewi, M. (1947). Acta ophthal., Kbh., 25, 47. 